\title{
A Finite-Difference Time-Domain Beam-Propagation Method for TE- and TM-Wave Analyses
}

\author{
Jun Shibayama, Member, IEEE, Member, OSA, Atsushi Yamahira, Takanori Mugita, \\ Junji Yamauchi, Member, IEEE, Member, OSA, and Hisamatsu Nakano, Fellow, IEEE
}

\begin{abstract}
The application of the existing time-domain beampropagation method (TD-BPM) based on the finite-difference (FD) formula has been limited to the TE-mode analysis. To treat the TM mode as well as the TE mode, an improved TD-BPM is developed using a low-truncation-error FD formula with the aid of the alternating-direction implicit scheme. To improve the accuracy in time, a Padé $(2,2)$ approximant is applied to the time axis. Although the truncation error in time is found to be $O\left(\Delta t^{2}\right)$, as in the case of the Padé $(1,1)$ approximant, this method allows us to use a large time step. A substantial reduction in CPU time is found when compared to the conventional method in which a broadly banded matrix is solved by the Bi-CGSTAB. The effectiveness in evaluating the TEand TM-mode waves is shown through the analysis of the power reflectivity from a waveguide facet. This method is also applied to the analysis of a waveguide grating. The accuracy and efficiency of the TD-BPM are assessed in comparison with the finite-difference time-domain method.
\end{abstract}

Index Terms-Finite-difference methods, optical beam propagation, optical waveguides, time-domain analysis, waveguide grating.

\section{INTRODUCTION}

A NALYSIS treating reflected waves is indispensable for modeling of many optical components and devices. Reflected waves can be treated by the finite-difference timedomain (FDTD) method [1]. Unfortunately, the FDTD method requires a small time step to fulfill the stability criterion. This increases the amount of computation for the analysis of an optically large structure.

To improve the efficiency in the time-domain analysis, considerable attention has been paid to the time-domain beam-propagation method (TD-BPM) [2], [3]. The feature of the TD-BPM is that the slowly varying envelope approximation (SVEA) is applied to the time axis, leading to a parabolic equation (a timedomain parabolic equation approach is also found in underwater acoustics [4]). The SVEA allows us to use an implicit scheme with a subsequent larger time step than that in the FDTD method. For the formulation of the TD-BPM, the finite-difference (FD) [3], [5]-[11] and the finite-element (FE) methods [12], [13] have mainly been used to discretize the space. Note that the application of the FD-based TD-BPMs developed so far has been limited only to the TE-mode analysis. The fact that the use of the alternating-direction implicit (ADI) scheme leads to high computational efficiency [10] encourages us to develop an FD-based TD-BPM for the TM-mode analysis.

Manuscript received December 9, 2002; revised March 14, 2003.

The authors are with Faculty of Engineering, Hosei University, Tokyo 1848584, Japan.

Digital Object Identifier 10.1109/JLT.2003.814392
For the BPM in a frequency domain, we have formulated modified FD formulas with second-order accuracy for both TE and TM modes [14], [15]. Subsequently, Chiou et al. have improved the modified FD formula, leading to the fourth-order accuracy with the aid of the generalized Douglas (GD) scheme for a two-dimensional (2-D) waveguide [16] (we refer to the formula as IFD4). Taking advantage of the ADI scheme, we have succeeded in applying the IFD4 to the analysis of a three-dimensional (3-D) waveguide, while maintaining a tridiagonal matrix [17], [18].

Note that the formulation of the 3-D BPM in the frequency domain corresponds to that of the 2-D TD-BPM. This fact motivates us to modify the 3-D BPM with the IFD4 to the TD-BPM. The modification is expected to allow the time-domain simulation of the TM mode as well as the TE mode with high accuracy.

In this paper, we develop an IFD4-based TD-BPM for the analysis of both TE and TM modes. To maintain the accuracy when using a large time step, we apply a Padé $(2,2)$ approximant [19], [9] to the time axis. So far, the truncation error in time for the TD-BPM has not been discussed in detail. Therefore, we derive the truncation errors for the Padé $(1,1)$ and $(2,2)$, paying attention to the use of the ADI scheme (comparison to the frequency-domain analysis is presented in Appendix B). Although the error of the Padé $(2,2)$ is found to be $O\left(\Delta t^{2}\right)$ as in the case of the Padé $(1,1)$, the amplitude of the error is smaller than that of the Padé $(1,1)$, allowing the use of a larger $\Delta t$. It is shown, through numerical examples of pulse propagation, that $\Delta t$ in the Padé $(2,2)$ can be chosen to be ten times as large as that in the Padé $(1,1)$. It is also demonstrated that the present method is more efficient than the method with the conventional Crank-Nicolson (CN) scheme, in which a broadly banded matrix is solved by the Bi-CGSTAB [20]. To check the validity of evaluating polarization dependence, we analyze the power reflectivity from a waveguide facet, in which the radiation waves are absorbed using the perfectly matched layer (PML) boundary condition. Numerical results for the TE and TM modes show excellent agreement with Vassallo's results [21]. Further consideration is given to the analysis of a waveguide grating. The spectral responses comparable to those from the FDTD method can be obtained by the TD-BPM with a reduction in CPU time.

\section{FORMULATION}

\section{A. Fourth-Order Accurate Finite-Difference Formula (IFD4)}

To make the discussion self-contained, we briefly describe the IFD4 that satisfies the boundary condition at the interface between two different media. We follow the procedure formulated 


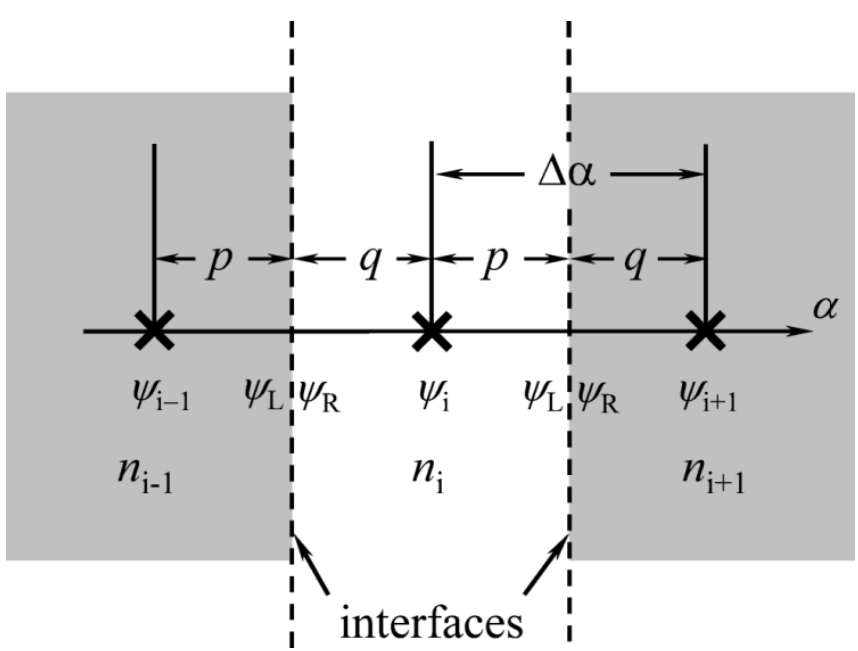

Fig. 1. Sampling points near the interface.

by the GD scheme [16], [18], in which the modified FD formulas [14], [15] have been extended to the fourth-order accuracy.

The sampling points near the interface are defined as shown in Fig. 1, in which $\alpha=x$ or $z$. Throughout this paper, we only consider the case where the interface lies midway between sampling points, i.e., $p=q=\Delta \alpha / 2$.

$\psi_{L}$ and $\psi_{R}$ must satisfy the following boundary conditions:

$$
\begin{gathered}
\psi_{L}=\psi_{R} \\
\theta \frac{\partial \psi_{L}}{\partial \alpha}=\frac{\partial \psi_{R}}{\partial \alpha}
\end{gathered}
$$

where

$$
\begin{array}{ll}
\psi=E_{y}, \theta=1 & \text { (for TE mode) } \\
\psi=H_{y}, \theta=\frac{n_{i+1}^{2}}{n_{i}^{2}} & \text { (for TM mode) }
\end{array}
$$

in which $n, E_{y}$, and $H_{y}$ represent the refractive index, electric, and magnetic fields, respectively. Using Taylor series expansions, $\psi_{L}$ and $\psi_{R}$ are expressed by the fields at the sampling points. Then, $\psi_{L}$ is connected with $\psi_{R}$ in accordance with the boundary conditions including up to the fifth derivative. Doing so leads to the following equation:

$$
\begin{aligned}
\psi_{i \pm 1}=\gamma_{0}^{ \pm} \psi_{i}+\gamma_{1}^{ \pm} \frac{\partial \psi_{i}}{\partial \alpha} & +\gamma_{2}^{ \pm} \frac{\partial^{2} \psi_{i}}{\partial \alpha^{2}}+\gamma_{3}^{ \pm} \frac{\partial^{3} \psi_{i}}{\partial \alpha^{3}} \\
& +\gamma_{4}^{ \pm} \frac{\partial^{4} \psi_{i}}{\partial \alpha^{4}}+\gamma_{5}^{ \pm} \frac{\partial^{5} \psi_{i}}{\partial \alpha^{5}}+O\left(\Delta^{6}\right) .
\end{aligned}
$$

The coefficients $\gamma_{i}(i=1, \ldots, 5)$ in (3) are presented in Appendix A. Equation (3) means that $\psi_{i+1}$ and $\psi_{i-1}$ are expanded in terms of $\psi_{i}$, in which the field discontinuity at the interface is taken into account.

Before deriving a fourth-order formula, we prepare the first and second derivatives with $O\left(\Delta^{2}\right)$. Using

$$
\psi_{i \pm 1}=\gamma_{0}^{ \pm} \psi_{i}+\gamma_{1}^{ \pm} \frac{\partial \psi_{i}}{\partial \alpha}+\gamma_{2}^{ \pm} \frac{\partial^{2} \psi_{i}}{\partial \alpha^{2}}+O\left(\Delta^{3}\right)
$$

we get

$$
\begin{aligned}
\frac{\partial \psi_{i}}{\partial \alpha} & =\frac{\gamma_{2}^{+} \psi_{i-1}+\left(\gamma_{0}^{+} \gamma_{2}^{-}-\gamma_{0}^{-} \gamma_{2}^{+}\right) \psi_{i}-\gamma_{2}^{-} \psi_{i+1}}{\gamma_{1}^{-} \gamma_{2}^{+}-\gamma_{1}^{+} \gamma_{2}^{-}}+O\left(\Delta^{2}\right) \\
& \equiv D_{\alpha} \psi_{i}
\end{aligned}
$$

and

$$
\begin{aligned}
\frac{\partial^{2} \psi_{i}}{\partial \alpha^{2}} & =\frac{\gamma_{1}^{+} \psi_{i-1}+\left(\gamma_{0}^{+} \gamma_{1}^{-}-\gamma_{0}^{-} \gamma_{1}^{+}\right) \psi_{i}-\gamma_{1}^{-} \psi_{i+1}}{\gamma_{2}^{-} \gamma_{1}^{+}-\gamma_{2}^{+} \gamma_{1}^{-}}+O\left(\Delta^{2}\right) \\
& \equiv D_{\alpha}^{2} \psi_{i} .
\end{aligned}
$$

Eliminating the first derivative in (3), we obtain

$$
\begin{aligned}
& \frac{\gamma_{1}^{+} \psi_{i-1}+\left(\gamma_{0}^{+} \gamma_{1}^{-}-\gamma_{0}^{-} \gamma_{1}^{+}\right) \psi_{i}-\gamma_{1}^{-} \psi_{i+1}}{\gamma_{2}^{-} \gamma_{1}^{+}-\gamma_{2}^{+} \gamma_{1}^{-}} \\
& =\frac{\partial^{2} \psi_{i}}{\partial \alpha^{2}}+g_{1} \frac{\partial^{3} \psi_{i}}{\partial \alpha^{3}}+g_{2} \frac{\partial^{4} \psi_{i}}{\partial \alpha^{4}}+g_{3} \frac{\partial^{5} \psi_{i}}{\partial \alpha^{5}}+O\left(\Delta^{4}\right)
\end{aligned}
$$

where $g_{1}, g_{2}$, and $g_{3}$ are defined in Appendix A. Since $g_{3}=0$ for $p=q=\Delta \alpha / 2$, the right-hand side of (7) reduces to

$$
\left(1+g_{1} \frac{\partial}{\partial \alpha}+g_{2} \frac{\partial^{2}}{\partial \alpha^{2}}\right) \frac{\partial^{2} \psi_{i}}{\partial \alpha^{2}}+O\left(\Delta^{4}\right) .
$$

The first and second derivatives in the parenthesis of (8) are replaced with (5) and (6), respectively. Since the left-hand side of (7) corresponds to (6), (8) can be written as follows:

$$
\frac{\partial^{2} \psi_{i}}{\partial \alpha^{2}} \simeq \frac{D_{\alpha}^{2} \psi_{i}}{\left(1+g_{1} D_{\alpha}+g_{2} D_{\alpha}^{2}\right)}+O\left(\Delta^{4}\right) \equiv \frac{D_{\alpha}^{2} \psi_{i}}{\tilde{D}_{\alpha}^{2}} .
$$

Equation (9) is referred to IFD4, which will be applied to the ADI scheme in the next section.

In this formulation, the interface has been assumed to lie midway between sampling points. Under this assumption, the structures to be treated in Sections III and IV are accurately modeled while maintaining $O\left(\Delta^{4}\right)$. For generic problems with an arbitrary interface, one may use a staircase approximation. Further study, however, shows that the formulation can be generalized to allow an arbitrary interface position [17], in which the coefficients' $\gamma$ s should be slightly modified, although the truncation error deteriorates into $O\left(\Delta^{3}\right)[18]$.

\section{B. Derivation of TD-BPM Equations Based on the ADI Scheme}

Using the time dependence of $\exp (j \omega t)$ and ignoring the temporal second derivative based on the SVEA, we start with the following basic equation:

$$
\frac{\partial \psi}{\partial t}=\zeta\left(\nabla_{x}^{2}+\nabla_{z}^{2}\right) \psi+\xi \psi \equiv P \psi
$$

where $\zeta=-j c^{2} /\left(2 \omega n^{2}\right), \xi=-j \omega / 2$

$$
\begin{array}{ll}
\nabla_{\alpha}^{2}=\frac{\partial^{2}}{\partial \alpha^{2}} & \text { (for TE mode) } \\
\nabla_{\alpha}^{2}=n^{2} \frac{\partial}{\partial \alpha}\left(\frac{1}{n^{2}} \frac{\partial}{\partial \alpha}\right) & \text { (for TM mode) }
\end{array}
$$

in which $\omega, n$, and $c$ are the centered frequency, the refractive index, and the speed of light in a vacuum, respectively. Equation (10) can be formally written as

$$
\psi(t+\Delta t)=\exp ([P] \Delta t) \psi(t)
$$

where $[P]$ represents the matrix of the finite-difference approximation to $P$. In the conventional method, the exponential function of (11) is approximated by the Padé $(1,1)$ approximant. The Padé $(1,1)$ approximant is identical to the $\mathrm{CN}$ scheme, which has the advantage of selecting a large time step $\Delta t$ without encountering a stability problem. However, a large $\Delta t$ often degrades the accuracy in time. In order to avoid this degradation, 
we apply the Padé $(2,2)$ approximant to the exponential function of (11)[19], [9].

The $(2,2)$ Padé approximant of (11) is given by

$$
\psi^{l+1}=\frac{1+\frac{1}{2}[P] \Delta t+\frac{1}{12}[P]^{2} \Delta t^{2}}{1-\frac{1}{2}[P] \Delta t+\frac{1}{12}[P]^{2} \Delta t^{2}} \psi^{l} .
$$

With the multistep method [22], (12) can be reduced to the following two-step algorithm:

$$
\psi^{l+k / 2}=\frac{1+a_{k}[P] \Delta t}{1-a_{k}[P] \Delta t} \psi^{l+(k-1) / 2} \text { for } k=1 \text { and } 2
$$

where $a_{k}=\left(3+(-1)^{k} \sqrt{3} j\right) / 12$. The superscript $l$ indicates the position along the time axis. Note that the direct application of (9) to (10) or (13) results in linear equations; each equation involves nine unknowns. As a result, the system of equations becomes a broadly banded matrix, which is usually solved by iterative techniques such as the Bi-CGSTAB. This is highly time-consuming, as will be seen later. Therefore, we employ the ADI scheme to maintain a tridiagonal matrix.

To solve (13) by the ADI scheme, we divide it as follows:

$$
\begin{aligned}
& {\left[1-a_{k} \Delta t\left(\zeta \nabla_{x}^{2}+\frac{\xi}{2}\right)\right]\left[1-a_{k} \Delta t\left(\zeta \nabla_{z}^{2}+\frac{\xi}{2}\right)\right] \psi^{l+k / 2}} \\
& =\left[1+a_{k} \Delta t\left(\zeta \nabla_{x}^{2}+\frac{\xi}{2}\right)\right]\left[1+a_{k} \Delta t\left(\zeta \nabla_{z}^{2}+\frac{\xi}{2}\right)\right] \psi^{l+(k-1) / 2} .
\end{aligned}
$$

Applying (9) to the second derivatives in (14), we have

$$
\begin{aligned}
& {\left[\left(1-\frac{a_{k} \Delta t \xi}{2}\right) \tilde{D}_{x}^{2}-a_{k} \Delta t \zeta D_{x}^{2}\right]} \\
& \times\left[\left(1-\frac{a_{k} \Delta t \xi}{2}\right) \tilde{D}_{z}^{2}-a_{k} \Delta t \zeta D_{z}^{2}\right] \psi^{l+k / 2} \\
& =\left[\left(1+\frac{a_{k} \Delta t \xi}{2}\right) \tilde{D}_{x}^{2}+a_{k} \Delta t \zeta D_{x}^{2}\right] \\
& \quad \times\left[\left(1+\frac{a_{k} \Delta t \xi}{2}\right) \tilde{D}_{z}^{2}+a_{k} \Delta t \zeta D_{z}^{2}\right] \psi^{l+(k-1) / 2} .
\end{aligned}
$$

Finally, we obtain the following equations:

$$
\begin{aligned}
& {\left[\left(1-\frac{a_{k} \Delta t \xi}{2}\right) \tilde{D}_{x}^{2}-a_{k} \Delta t \zeta D_{x}^{2}\right] \psi^{*}} \\
& \quad=\left[\left(1+\frac{a_{k} \Delta t \xi}{2}\right) \tilde{D}_{z}^{2}+a_{k} \Delta t \zeta D_{z}^{2}\right] \psi^{l+(k-1) / 2} \\
& {\left[\left(1-\frac{a_{k} \Delta t \xi}{2}\right) \tilde{D}_{z}^{2}-a_{k} \Delta t \zeta D_{z}^{2}\right] \psi^{l+k / 2}} \\
& \quad=\left[\left(1+\frac{a_{k} \Delta t \xi}{2}\right) \tilde{D}_{x}^{2}+a_{k} \Delta t \zeta D_{x}^{2}\right] \psi^{*} .
\end{aligned}
$$

It is worth mentioning that (16) and (17) maintain tridiagonal matrices, which can be solved by efficient techniques such as the Thomas algorithm.

In general, the formulation for the TM mode results in a nonunitary matrix, which may give rise to a numerical instability. This is often significant for a three-dimensional full-vectorial BPM in the frequency domain [23], [24]. Fortunately, we do not encounter any stability problem in the following analyses. One reason for this is that we only treat two-dimensional slab waveguides. In addition, the present
TD-BPM is based on the so-called semivectorial formulation that ignores mixed derivatives. This formulation may relieve the stability problem, when compared to the full-vectorial BPM in the frequency domain.

\section{Error Analysis in Time}

So far, no literature has investigated the truncation error in time for the TD-BPM. In this section, we check the truncation error in time, following the procedure described in [25] (comparison to the frequency-domain BPM is given in Appendix B). For the sake of simplicity, we investigate the case for the central FD approximation to the space derivatives; the truncation errors in space are $O\left(\Delta x^{2}\right)$ and $O\left(\Delta z^{2}\right)$. In the following, we denote the partial derivatives using the subscript notation, for instance, $\partial^{3} \psi / \partial t^{3} \equiv \psi_{t t t}$.

First, we present the truncation error of the Padé $(1,1)$ approximant. When (11) is approximated by the Padé $(1,1)$, which corresponds to the conventional $\mathrm{CN}$ scheme, the truncation error is derived as

$$
\epsilon^{(1,1)}=\Delta t^{2}\left[\frac{1}{24} \psi_{t t t}-\frac{\zeta}{8}\left(\psi_{x x t t}+\psi_{z z t t}\right)+\frac{\xi^{2}}{4} \psi_{t t}\right]=O\left(\Delta t^{2}\right) .
$$

Applying the ADI scheme to the Padé $(1,1)$ gives rise to the additional error, so that the truncation error results in

$$
\begin{aligned}
\epsilon_{\mathrm{ADI}}^{(1,1)} & =\epsilon^{(1,1)}+\zeta^{2} \frac{\Delta t^{2}}{4}\left[\psi_{x x z z t}+\frac{\xi}{2 \zeta}\left(\psi_{x x t}+\psi_{z z t}\right)+\frac{\xi^{2}}{4 \zeta^{2}} \psi_{t}\right] \\
& =O\left(\Delta t^{2}\right) .
\end{aligned}
$$

It can be said that the truncation error is $O\left(\Delta t^{2}\right)$ for the Pade $(1,1)$ approximant, regardless of the use of the ADI scheme.

For the Padé $(2,2)$ approximant, the truncation error is derived from (13) as

$$
\begin{aligned}
\epsilon^{(2,2)} & \simeq \frac{\Delta t^{4}}{1920} \psi_{t t t t t}-\zeta \frac{\Delta t^{2}}{96}\left(\Delta x^{2} \psi_{x x x x t t}+\Delta z^{2} \psi_{z z z z t t}\right) \\
& =O\left(\Delta t^{4}\right)+O\left(\Delta t^{2} \Delta x^{2}\right)+O\left(\Delta t^{2} \Delta z^{2}\right)
\end{aligned}
$$

Note that only the leading term is explicitly presented for errors of the order of $\Delta t^{4}$. It is seen that approximating the space derivatives by the FD formula yields truncation errors of $O\left(\Delta t^{2} \Delta x^{2}\right)$ and $O\left(\Delta t^{2} \Delta z^{2}\right)$ in addition to $O\left(\Delta t^{4}\right)$. For the ADI scheme, the following truncation error including the additional one is derived from (14):

$$
\begin{aligned}
\epsilon_{\mathrm{ADI}}^{(2,2)}= & \epsilon^{(2,2)}+\zeta^{2} \frac{\Delta t^{2}}{12}\left[\psi_{x x z z t}+\frac{\xi}{2 \zeta}\left(\psi_{x x t}+\psi_{z z t}\right)+\frac{\xi^{2}}{4 \zeta^{2}} \psi_{t}\right] \\
& +O\left(\Delta t^{3}\right) \\
= & O\left(\Delta t^{2}\right) .
\end{aligned}
$$

Consequently, the use of the ADI scheme with the Padé $(1,1)$ or $(2,2)$ induces the truncation error of $O\left(\Delta t^{2}\right)$. Notice that the amplitude of the additional error in (21) is just one-third of that of (19). However, the improvement in accuracy is more significant than that expected from the error analysis, as will be seen in Section III.

\section{AnAlysis of PUlse PROPAGation}

Studying the propagation of an optical pulse is essential in the time-domain analysis. To check the accuracy of the present 


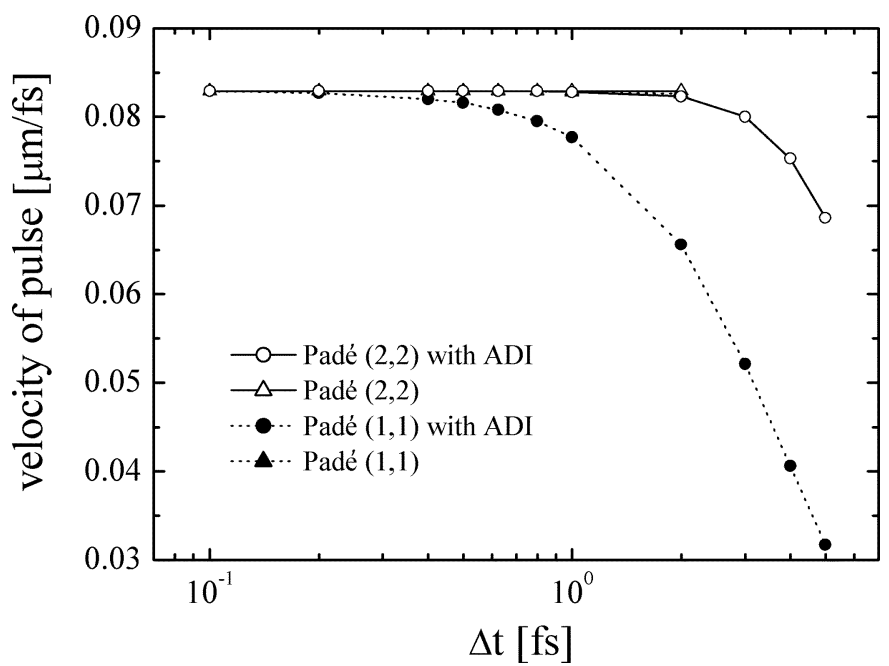

Fig. 2. Velocity of pulse as a function of $\Delta t$.

TD-BPM, we investigate the velocity of the pulse in a slab waveguide. The refractive indexes of the core and cladding are $n_{\mathrm{co}}=$ 3.60 and $n_{\mathrm{cl}}=3.24$, respectively, and the core is $0.262 \mu \mathrm{m}$ wide. A wavelength of $\lambda=0.86 \mu \mathrm{m}$ is used. The normalized frequency is fixed to be $V=1.5$, so that only the fundamental mode propagates. The input pulse consists of the eigenmode field in the $x$-direction and the Gaussian profile with a $1 / e$ full width of $4 \mu \mathrm{m}$ in the $z$-direction. The numerical parameters are as follows: the sampling widths are $\Delta x \simeq 0.013 \mu \mathrm{m}$ and $\Delta z=0.01 \mu \mathrm{m}$, and the number of sampling points are $N_{x}=140$ and $N_{y}=2000$ (due to the symmetry of the waveguide, only the half-region is analyzed).

Fig. 2 shows the velocity of the pulse for the TE mode as a function of $\Delta t$. For reference, the result obtained from the Padé $(1,1)$ approximant with the ADI scheme is included. In addition, the results obtained without the ADI scheme are presented in which a broadly banded matrix is solved by the Bi-CGSTAB. To obtain reasonable accuracy, we set the residual in norm to be $10^{-7}$. Note that the results without the ADI scheme are omitted for $\Delta t>2 \mathrm{fs}$, since the number of iteration is significantly increased, resulting in very slow convergence.

In the following discussion, we regard a velocity of $0.0829 \mu \mathrm{m} / \mathrm{fs}$ as a converged value, since the Padé $(2,2)$ without ADI gives a constant value of $0.0829 \mu \mathrm{m} /$ fs ranging from $\Delta t=0.1$ to 2 fs. It is seen in Fig. 2 that the Padé $(1,1) \mathrm{s}$ with and without the ADI scheme yield the converged values for $\Delta t=0.1$ and $1 \mathrm{fs}$, respectively. As found in (19), the difference between the two methods lies in the additional error that is caused by the ADI scheme. Therefore, the additional error generated from the ADI scheme for the Padé $(1,1)$ severely affects the accuracy, particularly for a large $\Delta t$. In contrast, the Padé $(2,2)$ s with and without the ADI scheme yield the converged values for $\Delta t=1$ and $2 \mathrm{fs}$, respectively. The contribution of the Padé $(2,2)$ toward maintaining the accuracy is significant when using the ADI scheme, although the comparison of (19) and (21) indicates that the amplitude of the additional error is only one-third of that of the Padé $(1,1)$. To explain this reason, we may have to perform the numerical dispersion analysis. Very recently, Lim et al. [11] have calculated the numerical

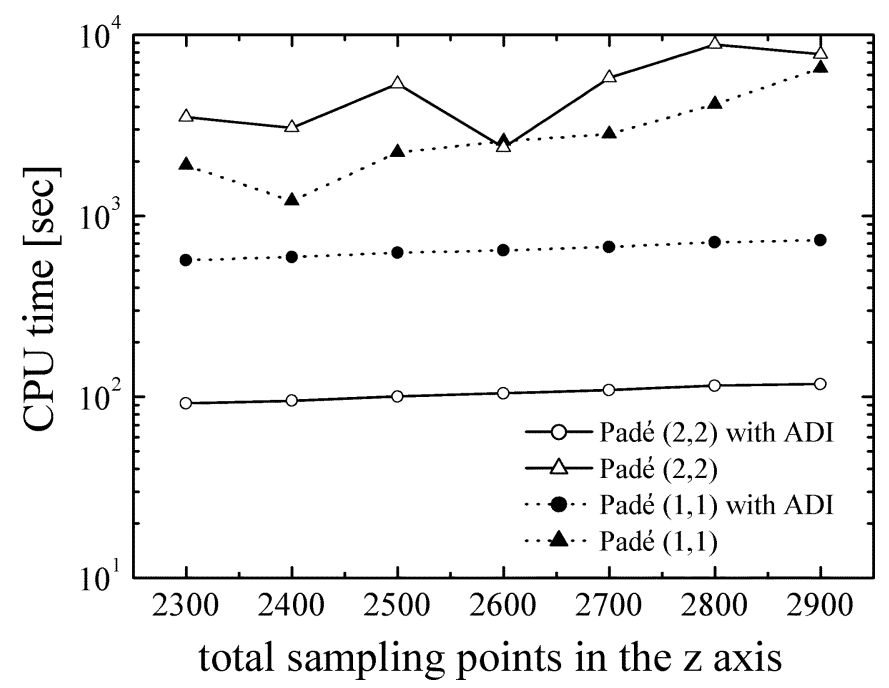

Fig. 3. CPU time needed for pulse propagation ( $t=10 \mathrm{fs}$ ).

dispersion of the TD-BPM, in which only a simple model has been investigated: a homogeneous free-space region in one dimension. Unfortunately, the numerical dispersion analysis for the present TD-BPM is not simple, since the method treats an inhomogeneous region in two dimensions and its formulation is based on the ADI scheme. The analysis is therefore beyond the scope of this paper and will be left for a future study.

Here we compare CPU times among the four methods. The time step $\Delta t$ is chosen to yield the converged value of the pulse velocity as discussed above. The calculations are carried out on an $850-\mathrm{MHz}$ PC. Fig. 3 shows the CPU time needed for pulse propagation ( $t=10 \mathrm{fs})$ as a function of sampling points in the $z$-axis. Note that the CPU time does not linearly increase when using the Bi-CGSTAB, while it monotonically increases when using the ADI scheme. It is worth mentioning that the use of the Padé $(2,2)$ with the ADI scheme leads to a substantial reduction in CPU time.

\section{ANALYSES of REFLECTION PRoblems}

\section{A. Waveguide Facet}

We analyze the facet reflectivity of an optical waveguide, which exhibits polarization dependence in a strongly guiding structure. The waveguide to be analyzed is the same as that used in Fig. 2, except that the end of the waveguide is terminated with air (see the inset of Fig. 4). In a previous paper [7], we calculated the power reflectivity using only the cross-section of the pulse in the transverse direction. However, the reflectivity in this calculation was found to be somewhat sensitive to the choice of $\Delta t$. To avoid this, we calculate

$$
P_{\mathrm{TE}}=\frac{\left|\int \psi_{\mathrm{in}} \psi_{\mathrm{ref}}^{*} d x d z\right|^{2}}{\left(\int\left|\psi_{\mathrm{in}}\right|^{2} d x d z\right)^{2}}
$$

for the TE mode, where $\psi_{\text {in }}$ and $\psi_{\text {ref }}$ represent the input and reflected fields, respectively. For the TM mode, we use the following formula similar to Vassallo's [27, (2)]:

$$
P_{\mathrm{TM}}=\frac{\left|\int n^{-2} \psi_{\mathrm{in}} \psi_{\mathrm{ref}}^{*} d x d z\right|^{2}}{\left(\int n^{-2}\left|\psi_{\mathrm{in}}\right|^{2} d x d z\right)^{2}}
$$




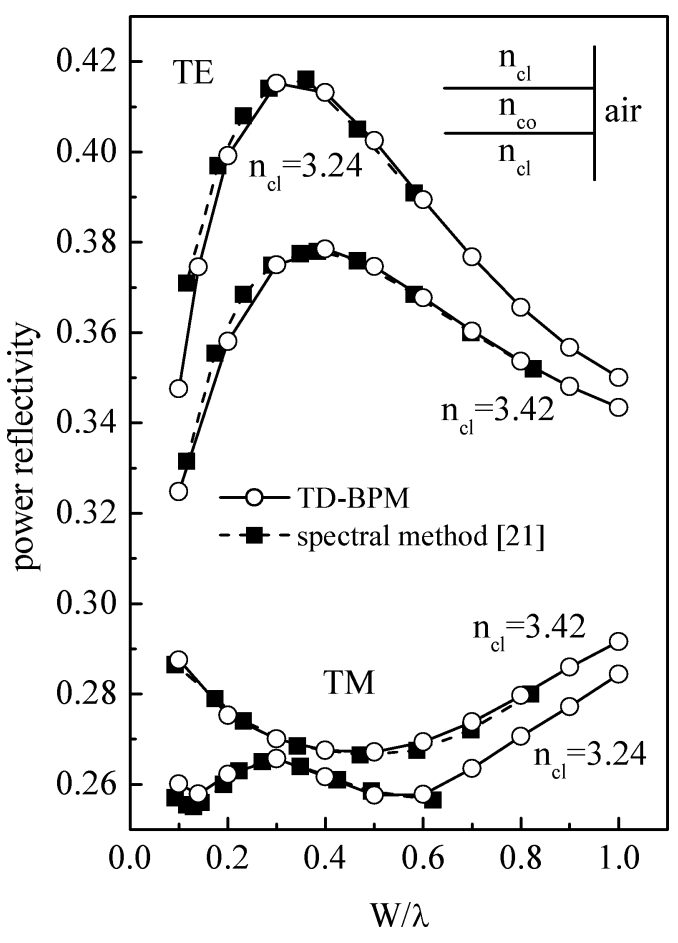

Fig. 4. Power reflectivity as a function of normalized core width.

In this structure, the radiation waves occur at the interface between the waveguide and the air region. To absorb the radiation waves, we apply the so-called coordinate scaling PML [26], which matches the ADI scheme. The PML parameters are chosen as a conductivity profile of $n=3$ and a theoretical reflection coefficient of $R(0)=10^{-6}$ for a 16-cell PML.

Fig. 4 shows the power reflectivity as a function of core width $W$ normalized to a wavelength of $\lambda=0.86 \mu \mathrm{m}$. A waveguide with $n_{\mathrm{cl}}=3.42$ is also investigated. The time step and the longitudinal sampling width are taken to be $\Delta t=1.0 \mathrm{fs}$ and $\Delta z=0.02 \mu \mathrm{m}$, respectively. The number of sampling points is 20 in the core. It is found that the results obtained from the TD-BPM agree well with those from the spectral method [21], demonstrating the validity of the TD-BPM for both TE- and TM-mode analyses.

Since the validity of the present TD-BPM has been verified for the problem with a single interface, consideration is given next to a problem with multiple interfaces.

\section{B. Waveguide Grating}

We analyze the waveguide grating shown in Fig. 5. The refractive indexes are $n_{\mathrm{co} 1}=3.24, n_{\mathrm{co} 2}=3.6$, and $n_{\mathrm{cl}}=2.0$. The grating period is $\Lambda_{+}=\Lambda_{-}=0.128 \mu \mathrm{m}$ and there are 20 periods in the core. This numerical example is chosen to show strong polarization dependence. In order to check the efficiency and the accuracy, we also analyze the same structure using the FDTD method.

For the multiple interface problem treated here, the forward and backward waves propagating toward the $\pm z$-directions mainly predominate the reflectivity characteristics. It follows that $\Delta z$ to be used is closely related to the accuracy of the numerical results. Therefore, we investigate how large a $\Delta z$

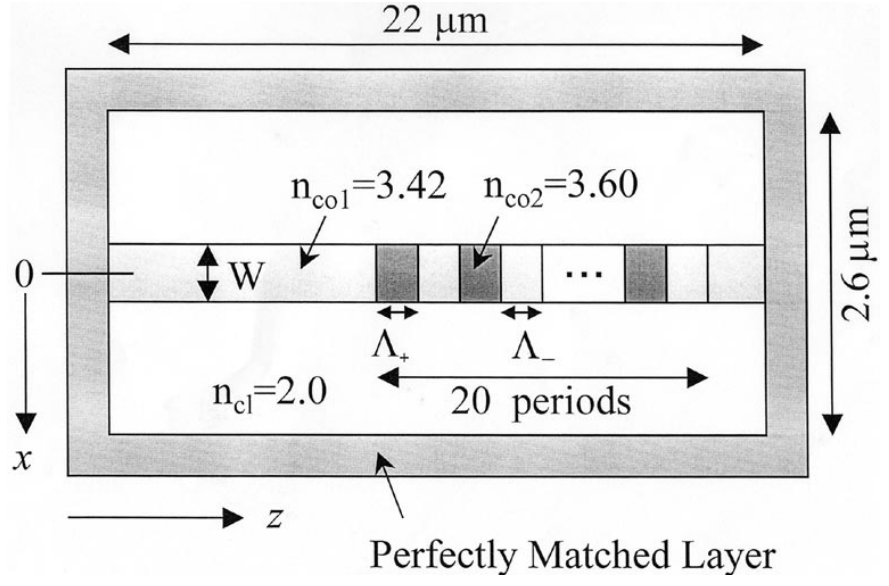

Fig. 5. Waveguide grating. The number of PML cells is taken to be 16 .

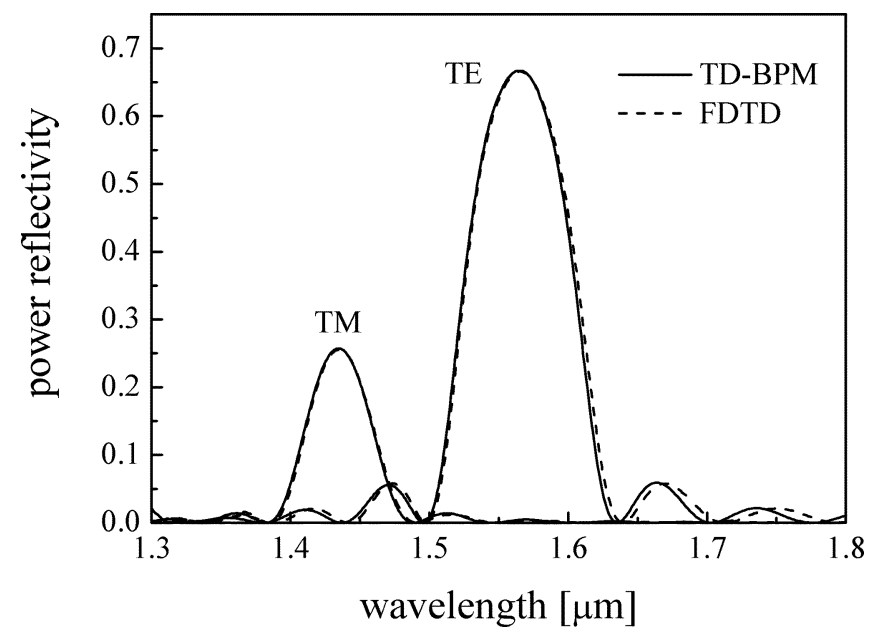

Fig. 6. Spectral response of the power reflectivity. The center wavelengths used in the calculation are chosen in such a way that they match the peak wavelengths, i.e., 1.55 and $1.44 \mu \mathrm{m}$ are used for the TE and TM modes, respectively.

can be used in this grating structure, with $\Delta x$ being fixed for both methods.

For the present TD-BPM, we use $\Delta t=1$ fs and $\Delta x=$ $W / 20 \simeq 0.013 \mu \mathrm{m}$ to calculate the spectral response of the grating. As a result, it is found that $\Delta z=0.064 \mu \mathrm{m}$ is sufficient to yield a converged value of the spectral response, in which one grating period is discretized by only four points. On the other hand, for the FDTD method, $\Delta z$ should be reduced to $0.0128 \mu \mathrm{m}$ to give a converged value. In this case, $\Delta t$ is reduced to $\simeq 0.03 \mathrm{fs}$ due to the stability criterion.

Fig. 6 shows the spectral response of the power reflectivity evaluated from the ratio between the discrete Fourier transforms of reflected pulse and the incident one, using the numerical parameters discussed above. The results of the TD-BPM are in close agreement with those of the FDTD method for both TE and TM modes, although the response for wavelengths larger than $1.65 \mu \mathrm{m}$ slightly deviates from that of the FDTD method. This deviation probably stems from the fact that the temporal second derivative is omitted in the TD-BPM. It is noteworthy, nevertheless, that the CPU time for the present TD-BPM is reduced to $30 \%$ of that for the FDTD method. 


\section{CONCLUSION}

A TD-BPM based on the fourth-order accurate finite-difference formula has been developed, which allows us to simulate the waves of the TM mode as well as the TE mode. The use of the Padé $(2,2)$ approximant with the ADI scheme leads to efficient calculations while maintaining high accuracy. To check the validity of the method, we analyze the facet reflectivity of the slab waveguides with polarization dependence. The results for the TE and TM modes agree well with those obtained from the spectral method. Further consideration is given to the analysis of a waveguide grating. The present TD-BPM efficiently provides spectral response comparable to the FDTD method.

Although the calculation in this paper is restricted to the case where the interface lies midway between sampling points, this method can be tailored to treat a general position of the interface [17]. This enables us to analyze more complicated structures.

\section{APPENDIX A \\ COEFFICIENTS USED IN THE IFD4}

The coefficients used in (3) are as follows:

$$
\begin{aligned}
\gamma_{0}^{ \pm}=1+ & \frac{q^{2} \eta^{ \pm}}{2}+\frac{q^{4}\left(\eta^{ \pm}\right)^{2}}{24}+O\left(\Delta^{6}\right) \\
\gamma_{1}^{ \pm}= & \left\{p+\frac{p q^{2} \eta^{ \pm}}{2}+\frac{p q^{4}\left(\eta^{ \pm}\right)^{2}}{24}\right. \\
& \left.+\theta^{ \pm}\left(q+\frac{q^{3} \eta^{ \pm}}{6}+\frac{q^{5}\left(\eta^{ \pm}\right)^{2}}{120}\right)\right\}+O\left(\Delta^{6}\right) \\
\gamma_{2}^{ \pm}= & \frac{p^{2}}{2}+\frac{q^{2}}{2}+\frac{p^{2} q^{2} \eta^{ \pm}}{4}+\frac{q^{4} \eta^{ \pm}}{12} \\
& +\theta^{ \pm}\left(p q+\frac{p q^{3} \eta^{ \pm}}{6}\right)+O\left(\Delta^{6}\right) \\
\gamma_{3}^{ \pm}= & \pm \frac{p^{3}}{6}+\frac{p q^{2}}{2}+\frac{p^{3} q^{2} \eta^{ \pm}}{12}+\frac{p q^{4} \eta^{ \pm}}{12} \\
& \left.+\theta^{ \pm}\left(\frac{p^{2} q}{2}+\frac{q^{3}}{6}+\frac{p^{2} q^{3} \eta^{ \pm}}{12}+\frac{q^{5} \eta^{ \pm}}{60}\right)\right\}+O\left(\Delta^{6}\right) \\
\gamma_{4}^{ \pm}= & \frac{p^{4}}{24}+\frac{p^{2} q^{2}}{4}+\frac{q^{4}}{24}+\theta^{ \pm}\left(\frac{p^{3} q}{6}+\frac{p q^{3}}{6}\right)+O\left(\Delta^{6}\right) \\
\gamma_{5}^{ \pm}= & \pm\left\{\frac{p^{5}}{120}+\frac{p^{3} q^{2}}{12}+\frac{p q^{4}}{24}+\theta^{ \pm}\left(\frac{p^{4} q}{24}+\frac{p^{2} q^{3}}{12}+\frac{q^{5}}{120}\right)\right\} \\
& +O\left(\Delta^{6}\right)
\end{aligned}
$$

where

$$
\theta^{ \pm}= \begin{cases}1, & (\text { for TE mode }) \\ \frac{n_{i \pm 1}^{2}}{n_{i}^{2}}, & (\text { for TM mode })\end{cases}
$$

and $\eta^{ \pm}=k_{0}^{2}\left(n_{i}^{2}-n_{i \pm 1}^{2}\right)$, in which $k_{0}=2 \pi / \lambda$.

The coefficients used in (7) are expressed as

$$
\begin{aligned}
& g_{1}=\frac{\gamma_{3}^{-} \gamma_{1}^{+}-\gamma_{3}^{+} \gamma_{1}^{-}}{\gamma_{2}^{-} \gamma_{1}^{+}-\gamma_{2}^{+} \gamma_{1}^{-}} \\
& g_{2}=\frac{\gamma_{4}^{-} \gamma_{1}^{+}-\gamma_{4}^{+} \gamma_{1}^{-}}{\gamma_{2}^{-} \gamma_{1}^{+}-\gamma_{2}^{+} \gamma_{1}^{-}} \\
& g_{3}=\frac{\gamma_{5}^{-} \gamma_{1}^{+}-\gamma_{5}^{+} \gamma_{1}^{-}}{\gamma_{2}^{-} \gamma_{1}^{+}-\gamma_{2}^{+} \gamma_{1}^{-}} .
\end{aligned}
$$

\section{APPENDIX B}

\section{COMPARISON OF THE ADDITIONAL ERRORS OF THE TIME-DOMAIN AND FREQUENCY-DOMAIN BPMS}

For the TD-BPM with the Padé $(1,1)$ approximant, the use of the ADI scheme degrades the accuracy, particularly for a large $\Delta t$, as discussed in Section III. Therefore, the Padé $(2,2)$ is required to maintain the accuracy. On the other hand, for the frequency-domain BPM, the use of the ADI scheme hardly affects the accuracy in the Padé $(1,1)$ approximant [18]. Here, we compare the additional errors generated by the ADI scheme between the TD and frequency-domain BPMs.

For the Padé $(1,1)$ in the frequency-domain BPM, the additional error is written as

$$
\epsilon=\frac{\Delta z^{2}}{4 \rho^{2}}\left[\phi_{x x y y z}+\frac{\nu}{2}\left(\phi_{x x z}+\phi_{y y z}\right)+\frac{\nu^{2}}{4} \phi_{z}\right]
$$

where $\rho=2 j k_{0} n_{0}$ and $\nu=k_{0}^{2}\left(n^{2}-n_{0}^{2}\right)$, in which $n_{0}$ is the reference refractive index.

The coefficient of (24) can be rewritten as $\Delta z^{2} / 4 \rho^{2}=$ $-\Delta z^{2} / 16 k_{0}^{2} n_{0}^{2}$. For the TD-BPM, the coefficient of the additional error in (19) can be rewritten as $\Delta t^{2} \zeta^{2} / 4=$ $-\Delta z^{2} / 16 k_{0}^{2} n^{2}$. It can be seen that these two coefficients are identical for $n_{0}=n$. Therefore, we focus our attention on the terms in the parentheses.

We compare $\nu$ in (24) with $\xi / \zeta$ in (19). Notice that $\nu=$ $k_{0}^{2}\left(n^{2}-n_{0}^{2}\right)$ can be regarded as a small value, since $n_{0}$ is generally chosen to be close to $n$. This leads to the fact that $\nu$ is smaller than $\xi / \zeta\left(=k_{0}^{2} n^{2}\right)$.

In addition, the derivatives in (24) may be smaller than those in (19), since the field in the frequency-domain analysis, which only treats a forward propagating field, is expected to move more slowly than that in the TD analysis. It follows that the additional error in the frequency-domain BPM is smaller than that in the TD-BPM. This is the reason why the additional error in the frequency-domain BPM hardly affects the numerical results.

\section{ACKNOWLEDGMENT}

The authors would like to thank T. Murata for valuable discussions on the IFD4.

\section{REFERENCES}

[1] S. T. Chu, W. P. Huang, and S. K. Chaudhuri, "Simulation and analysis of waveguide based optical integrated circuits," Comput. Phys. Commun., vol. 68, pp. 451-484, 1991.

[2] M. Scalora and M. E. Crenshaw, "A beam propagation method that handles reflections," Opt. Commun., vol. 108, pp. 191-196, 1994.

[3] P. L. Liu, Q. Zhao, and F. S. Choa, "Slow-wave finite-difference beam propagation method," IEEE Photon. Technol. Lett., vol. 7, no. 8, pp. 890-892, 1995.

[4] J. E. Murphy, "Finite-difference treatment of a time-domain parabolic equation: Theory," J. Acoust. Soc. Amer., vol. 77, no. 5, pp. 1958-1960, 1985 .

[5] Y. Yuan, R. Jambunathan, J. Singh, and P. Bhattacharya, "Finite-difference time-domain analysis and experimental examination of the performance of a coupled-cavity MQW laser/active waveguide at $1.54 \mu \mathrm{m}$," IEEE J. Quantum Electron., vol. 33, no. 3, pp. 408-415, 1997.

[6] G. H. Jin, J. Harari, J. P. Vilcot, and D. Decoster, "An improved timedomain beam propagation method for integrated optics components," IEEE Photon. Technol. Lett., vol. 9, no. 3, pp. 348-350, 1997.

[7] J. Shibayama, T. Takahashi, J. Yamauchi, and H. Nakano, "Finite-difference time-domain beam propagation method for analysis of threedimensional optical waveguides," Electron. Lett., vol. 35, no. 18, pp. $1548-1549,1999$. 
[8] J. Yamauchi, J. Shibayama, and H. Nakano, "Application of the generalized Douglas scheme to optical waveguide analysis," Opt. Quantum Electron., vol. 31, no. 9/10, pp. 675-687, 1999.

[9] J. Shibayama, T. Takahashi, J. Yamauchi, and H. Nakano, "Time-domain finite-difference BPM with Padé approximants in time axis for analysis of circularly symmetric fields," Electron. Lett., vol. 36, no. 4, pp. 319-321, 2000

[10] 1 , "Efficient time-domain finite-difference beam propagation methods for the analysis of slab and circularly symmetric waveguides," J. Lightwave Technol., vol. 18, no. 3, pp. 437-442, 2000.

[11] J. J. Lim, T. M. Benson, E. C. Larkins, and P. Sewell, "Wideband finite-difference-time-domain beam propagation method," Microwave Opt. Technol. Lett., vol. 34, no. 4, pp. 243-247, 2002

[12] M. Koshiba, Y. Tsuji, and M. Hikari, "Time-domain beam propagation method and its application to photonic crystal circuits," J. Lightwave Technol., vol. 18, no. 1, pp. 102-110, 2000.

[13] V. F. Rodrígez-Esquerre and H. E. Hernández-Figueroa, "Novel timedomain step-by-step scheme for integrated optical applications," IEEE Photon. Technol. Lett., vol. 13, no. 4, pp. 311-313, 2001.

[14] J. Yamauchi, M. Sekiguchi, O. Uchiyama, J. Shibayama, and H. Nakano, "Modified finite-difference formula for the analysis of semivectorial modes in step-index optical waveguides," IEEE Photon. Technol. Lett., vol. 9, no. 7, pp. 961-963, 1997.

[15] J. Yamauchi, G. Takahashi, and H. Nakano, "Modified finite-difference formula for semivectorial $H$-field solutions of optical waveguides," IEEE Photon. Technol. Lett., vol. 10, no. 8, pp. 1127-1129, 1998

[16] Y. Chiou, Y. Chiang, and H. Chang, "Improved three-point formulas considering the interface conditions in the finite-difference analysis of step-index optical devices," J. Lightwave Technol., vol. 18, no. 2, pp. 243-251, 2000

[17] J. Yamauchi, T. Murata, and H. Nakano, "Semivectorial $H$-field analysis of rib waveguides by a modified beam-propagation method based on the generalized Douglas scheme," Opt. Lett., vol. 25, no. 24, pp. 1771-1773, 2000.

[18] — "Analysis of rib waveguides by a fourth-order accurate finite-difference beam-propagation method," IEICE Trans., vol. J85-C, no. 4, pp. 241-248, 2002

[19] Y. Chiou and H. Chang, "Efficient beam-propagation method based on Padé approximants in the propagation direction," Opt. Lett., vol. 22, no. 13, pp. 949-951, 1997.

[20] H. A. Van Der Vorst, "Bi-CGSTAB: A fast and smoothly converging variant of Bi-CG for the solution of nonsymmetric linear system," SIAM J. Sci. Statist. Comput., vol. 13, no. 2, pp. 631-644, 1992.

[21] C. Vassallo, "Reflectivity of multidielectric coatings deposited on the end facet of a weakly guiding dielectric slab waveguide," J. Opt. Soc. Amer. A, vol. 5, no. 11, pp. 1918-1928, 1988

[22] G. R. Hadley, "Multistep method for wide-angle beam propagation," Opt. Lett., vol. 17, no. 24, pp. 1743-1745, 1992.

[23] D. Yevick, J. Yu, W. Bardyszewski, and M. Glasner, "Stability issues in vector electric field propagation," IEEE Photon. Technol. Lett., vol. 7, no. 6, pp. 658-660, 1995.

[24] D. Yevick, "The application of complex Padé approximants to vector field propagation," IEEE Photon. Technol. Lett., vol. 12, no. 12, pp. $1636-1638,2000$

[25] K. W. Morton and D. F. Mayers, Numerical Solution of Partial Differential Equations: Cambridge Univ. Press, 1994, ch. 2-3.

[26] C. M. Rappaport, "Perfectly matched absorbing boundary conditions based on anisotropic lossy mapping of space," IEEE Microwave Guided Wave Lett., vol. 5, no. 3, pp. 90-92, 1995.

[27] C. Vassallo, "Polarization-independent antireflection coatings for semiconductor optical amplifiers," Electron. Lett., vol. 24, no. 1, pp. 61-62, 1988.

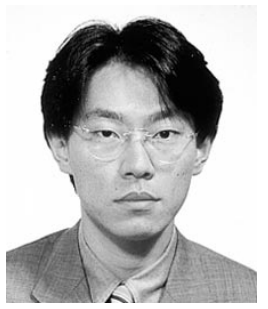

Jun Shibayama (M'03) was born in Chiba, Japan, on July 1 , 1969. He received the B.E., M.E., and Dr.E. degrees from Hosei University, Tokyo, Japan, in 1993, 1995, and 2001 respectively.

In 1995, he joined Opto-Technology Laboratory, Furukawa Electric Co., Ltd., Chiba, Japan. Since 1999, he has been an Assistant, Hosei University. His research interests include numerical analysis of optical waveguides.

Dr. Shibayama is a Member of the Optical Society of America (OSA) and the Institute of Electronics, Information and Communication Engineers (IEICE) of Japan.

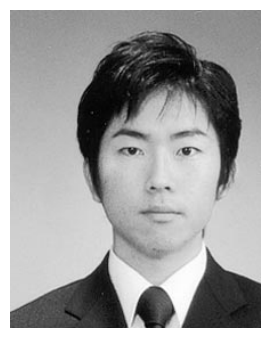

Atshushi Yamahira was born in Tochigi, Japan, on November 26, 1978. He received the B.E. degree from Hosei University, Tokyo, Japan, in 2001 and is currently pursuing the M.E. degree.

Mr. Yamahira is a Member of the Institute of Electronics, Information and Communication Engineers (IEICE) of Japan.

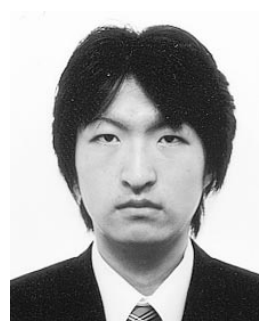

Takanori Mugita was born in Saitama, Japan, on May 28, 1979. He is working toward the B.E. degree at Hosei University, Tokyo, Japan.

Mr. Mugita is a Member of the Institute of Electronics, Information and Communication Engineers (IEICE) of Japan.

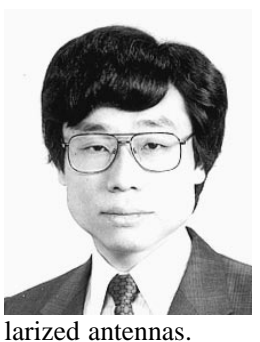

Junji Yamauchi (M'84) was born in Nagoya, Japan, on August 23, 1953. He received the B.E., M.E., and Dr.E. degrees from Hosei University, Tokyo, Japan, in 1976, 1978, and 1982, respectively. From 1984 to 1988, he served as a Lecturer in the Electrical Engineering Department of Tokyo Metropolitan Technical College. Since 1988, he has been a Member of the Faculty of Hosei University, where he is now a Professor of Electronic Informatics. His research interests include optical waveguides and circularly po-

Dr. Yamauchi is a Member of the Optical Society of America (OSA) and the Institute of Electronics, Information and Communication Engineers (IEICE) of Japan.

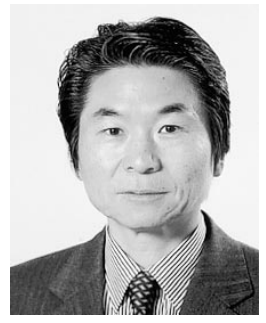

Hisamatsu Nakano (M'75-SM'87-F'92) was born in Ibaraki, Japan, on April 13, 1945. He received the B.E., M.E., and Dr.E. degrees in electrical engineering from Hosei University, Tokyo, Japan, in 1968, 1970, and 1974, respectively.

Since 1973, he has been a Member of the Faculty, Hosei University, where he is now a Professor of Electronic Informatics. He was a Visiting Associate Professor at Syracuse University, Syracuse, NY, from May to September 1981, a Visiting Professor at University of Manitoba, Canada, from March to September 1986, and a Visiting Professor at the University of California, Los Angeles, from September 1986 to March 1987. He has published more than 160 refereed journal papers and 120 international symposium papers on antenna and relevant problems. He is the author of Helical and Spiral Antennas (New York: Research Studies Press, Wiley, 1987). He published the chapter "Antenna Analysis Using Integral Equations," in Analysis Methods of Electromagnetic Wave Problems, vol. 2 (Norwood, MA: Artech House, 1996). His research topics include numerical methods for antennas, electromagnetic wave-scattering problems, and lightwave problems.

Dr. Nakano received an International Scientific Exchange Award from the Natural Sciences and Engineering Research Council of Canada. In 1987, he received the Best Paper Award from the Insitute of Electrical Engineers 5th International Conference on Antennas and Propagation. In 1994, he received the IEEE AP-S Best Application Paper Award (H. A. Wheeler Award). He is an Associate Editor of IEEE ANTENNAS AND PROPAGATION. He was a Member of the AP-S administrative committee in 2002. 